\title{
An adaptive cue combination model of human spatial reorientation
}

\begin{abstract}
Previous research has proposed an adaptive cue combination view of the development of human spatial reorientation (Newcombe \& Huttenlocher, 2006), whereby information from multiple sources is combined in a weighted fashion in localizing a target, as opposed to being modular and encapsulated (Hermer \& Spelke, 1996). However, no prior work has formalized this proposal and tested it against existing empirical data. We propose a computational model of human spatial reorientation that is motivated by probabilistic approaches to optimal perceptual cue integration (e.g. Ernst \& Banks, 2002) and to spatial location coding (Huttenlocher, Hedges, \& Duncan, 1991). We show that this model accounts for data from a variety of human reorientation experiments, providing support for the adaptive combination view of reorientation.
\end{abstract}

\section{Introduction}

The spatial world provides many cues to where things are. For example, a pirate may have buried a treasure chest five paces east from a distinctive tree and one hundred paces away from the shore. Locating the treasure often requires combining the various cues to locating the treasure in a probabilistic fashion, using appropriate weightings (Huttenlocher et al., 1991; Cheng, Shettleworth, Huttenlocher, \& Rieser, 2007). Combining cues allows for reduction of uncertainty concerning encoding and memory for individual cues, each of which might not be sufficiently informative in isolation, but which can jointly provide more precise, if sometimes biased, localization of a target. This view of perception and memory is also seen in the literature on perceptual cue integration (Ernst \& Banks, 2002; Jacobs, 2002; Knill \& Pouget, 2004; Berniker \& Kording, 2011). It is typically formulated in terms of probabilistic inference, which provides a rational account of human behavior under uncertainty. Such a probabilistic approach has recently

(C) 2017. This manuscript version is made available under the Elsevier user license http://www.elsevier.com/open-access/userlicense/1.0/ 
begun to be a focus of navigation research, especially in studies of how egocentric and allocentric systems interact with each other (e.g., Waisman, Lucas, Griffiths, \& Jacobs, 2011; Sjolund, Kelly, \& Mcnamara, 2014; Zhao \& Warren, 2015). But it has not been formally specified in explaining human behavior in spatial reorientation, an area in which there have been high-profile claims of modularity and information encapsulation, to which the cue-combination view provides an important alternative. Here we address this gap by formalizing cue combination in probabilistic terms and testing it against data on the development of spatial reorientation in human children and adults.

Research on behavior when organisms are disoriented (and therefore when egocentric spatial cues are not useful) seemed initially to support modularity, because geometric cues were used while potentially useful featural cues were not, both by rats and by young children (Gallistel, 1990; Hermer \& Spelke, 1994, 1996). The classic experiments were conducted in a rectangular room, in which the relative length of the walls defines two pairs of congruent corners (i.e., long wall to the left of short wall, or vice versa). Searches were directed to the correct corners as defined by geometry, but the addition of a feature such as one colored wall did not lead participants to narrow the choice to the correct corner. Although human adults do use featural cues, Hermer-Vazquez, Spelke \& Katsnelson (1999) argued that they do so only because human language allows for the combination of the output of different processing modules, a combination that they argued would not be possible without language. In this view, young children and non-human species share an ancestral geometric module for reorientation, later punctured by spatial language.

The modularity hypothesis has attracted much attention. But it has become clear that it cannot account for many aspects of the expanding data set on human reorientation and its development. One prominent problem is the room-size effect. Geometry is more likely to be used in small spaces and features are more likely to be used in large spaces, for children (Learmonth, Newcombe, \& Huttenlocher, 2001; Learmonth, Nadel, \& Newcombe, 2002), adults (Ratliff \& Newcombe, 2008b), fish (Sovrano, Bisazza, \& Vallortigara, 2007), chicks (Chiandetti, Regolin, Sovrano, \& Vallortigara, 2007; Sovrano \& Vallortigara, 2006; Vallortigara, Feruglio, \& Sovrano, 2005), and pigeons (Kelly, Spetch, \& Heth, 1998). In addition, short-term experience with the usefulness of a featural cue changes the behavior of young children (Twyman, Friedman, \& Spetch, 2007), human adults (Ratliff \& Newcombe, 2008a) and pigeons (Kelly \& Spetch, 2004). Further, rearing environment changes weighting of geometry and features, at least for convict fish (Brown, Spetch, \& Hurd, 2007) and mice (Twyman, Newcombe, \& Gould, 2013), 
although not chicks (Chiandetti \& Vallortigara, 2008, 2010). Cheng (2008) suggested abandoning a modularity approach.

Other non-modular approaches to the development of human reorientation have been proposed besides adaptive combination; for an overview, see Cheng, Huttenlocher, and Newcombe (2013). One computationally-specified non-modular approach uses an associative learning model (Miller, 2009), based on a model originally formulated to explain reorientation data from non-human animals (Miller \& Shettleworth, 2007) to explain findings from humans. In this account, cues compete with each other by gaining or losing strength based on a variant of the Rescorla-Wagner (1972) learning rule, adapted to encompass operant learning as well as classical conditioning. This model has the great virtue of precision in its assumptions about encoding and processing, and it provides a good fit to a variety of data. However, the extension of the original model to encompass human development relies on age-related variations in learning rate, an assumption that does not fit the developmental findings (Cheng, Huttenlocher, \& Newcombe, 2013). Studies with children involve very few trials (often just 4), and do not find better performance on the last trial than the first.

An alternative is to link the development of human reorientation to the development of cue combination, an idea suggested previously (e.g. Newcombe \& Huttenlocher, 2006) but not computationally specified or evaluated (Cheng et al., 2013). The purpose of this paper is to specify such a computational model, and compare it with the modular encapsulation-plus-language model. We also compare it to the associative model, using the same set of data examined by Miller (2009). We evaluate the generality of its explanatory power by cross-predicting independent sets of empirical data. We restrict our scope of investigation in this paper to the development of reorientation in humans, and caution that it remains to be determined whether the model also captures the behavior of non-human species in the reorientation paradigm, for whom operant learning may be more essential (Miller \& Shettleworth, 2007, 2008).

Table 1 summarizes the main differences between the proposed model, the modular hypothesis and the associative learning model of development. First, our model is grounded in the principle of cue combination as a form of probabilistic inference, suggesting that integration of information can occur from early in human development (i.e. it does not depend on language to bridge between otherwise hypothetically encapsulated modules). Second, our model does not require (although it can accommodate) a process of learning, since it is responsive to internal uncertainty based on perception and memory processes; in comparison, in the modular account, language learning is critical, as it then allows for 
modules to be linked, and in the associative model, learning based on external feedback is central to the reinforcement and suppression of cues. Third, our model includes a potential role of spatial language as a distinct cue that can exert an effect, but situates this effect against a background of cue combination. Thus, the inclusion of language as a strategic cue for reorientation differs from the position of modularity theory (Hermer \& Spelke, 1996; Hermer-Vazquez, 1997), in that we suggest that language is not the only way that information can be combined during reorientation; rather, it acts as an independent cue that helps to reduce uncertainty in reorientation. Fourth, we use a relatively small set of cues and minimal free parameters. The associative model has a higher number of cues and adjustable parameters, due to the fact that it also parameterizes the learning process. Finally, we use a combination of fitting and cross-prediction to evaluate the models, which provides a general, rigorous way of assessing model performances. In the following sections, we show that this simple proposed cue combination model accounts for existing empirical data.

\begin{tabular}{l|l|l|l} 
Property & Modularity hypothesis & Associative model & Cue combination model \\
\hline \hline Grounding principle & Encapsulation+language & Associative learning & Probabilistic inference \\
Role of learning & Language only & Central & Not required for humans \\
Role of language & Dominant once acquired & Unaccounted for & Strategic cue \\
Number of free parameters & Underspecified & Relatively high & Relatively low \\
Method of evaluation & Empirical & Fitting & Fitting+cross prediction
\end{tabular}

Table 1: Qualitative comparison of cue-combination and existing accounts of spatial reorientation.

The rest of this paper is organized as follows. We first illustrate the idea of cue combination in informal terms. We then present our computational model, which formalizes these ideas. We then describe the sources of empirical data on which we draw, and present three case studies in which we test our model against these empirical findings, and compare the results to those of alternative models.

\section{Illustration of cue combination}

Figure 1 illustrates the overall concept of cue combination. Here and elsewhere, we assume that a person is inside a closed space (e.g. a room), has seen a target object being hidden in one of a finite number of 
possible locations within that space (e.g. one of the corners of a room), and is then disoriented within that space. Their task is to recover the target object after disorientation. For illustrative purposes, suppose that there are two independent cues in this reorientation task. Each cue provides some information about the location of the target $t^{*}$, which is fixed and located in one of four possible locations. The height of the bars for the individual cues represents the strength of each cue at each location, which varies across the two cues. The taller bars correspond to locations that a cue strongly predicts to be possible target locations. The shorter bars correspond to non-target locations. Concretely, Cue 1 is a cue based on surface geometry of the enclosure that predicts corners $L_{2}$ and $L_{4}$ as the most likely candidates for target location, because these two corners are geometrically equivalent to the actual target location $\left(L_{2}\right)$. Cue 2 relies on an explicit landmark - a wall painted blue in this case - that predicts its adjacent corners $L_{2}$ and $L_{3}$ ambiguously as probable target locations, by virtue of association. Although both cues provide some degree of information in determining the location of the target, neither is sufficient to predict the target precisely. Thus, for a rational agent, an optimal strategy would be to combine information from the two cues, which would yield a substantially sharper response over the true target and hence allow for reduction of uncertainty - reflected in a resulting distribution of choice probabilities that peaks at the target location. 
a)

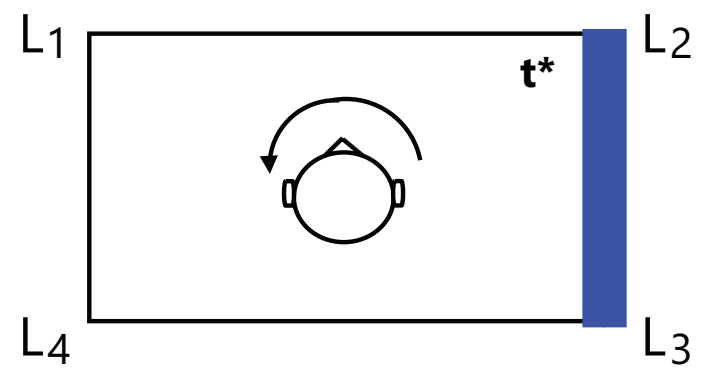

b)

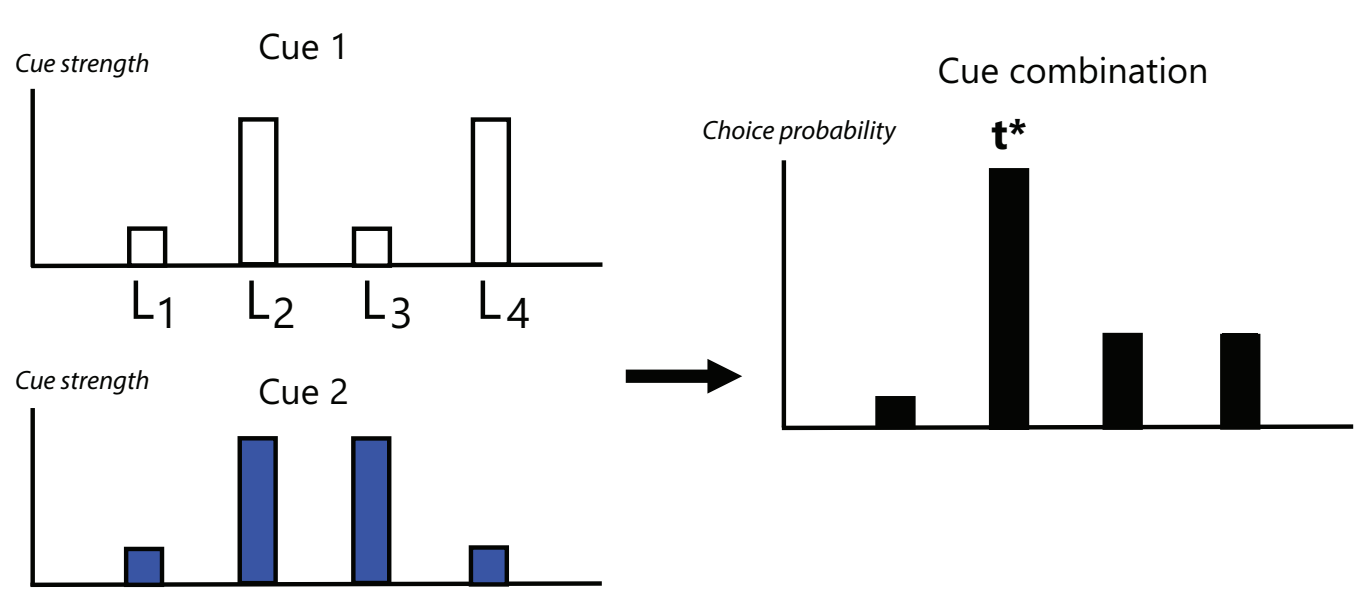

Figure 1: Illustration of cue combination in spatial reorientation. a) Target $t^{*}$ is located at one of four discrete locations $L_{1}, L_{2}, L_{3}, L_{4}$ in an enclosure (birds eye view), shown initially to and subject to retrieval by a person in the center of the enclosure who has been disoriented. b) Two independent cues each provide some information concerning the location of the target, but neither is sufficient to locate it fully. Cue 1 is based on surface geometry of the enclosure, and predicts $L_{2}$ and $L_{4}$ as the most probable locations of the target (due to their geometrical equivalence under rotation). Cue 2 relies on a landmark - a colored wall represented by the thick line on the right of the room in panel (a) - that predicts its adjacent corners $L_{2}$ and $L_{3}$ as probable locations for target. Although neither cue is fully predictive of the target location, combining information from the two cues (shown as black bars) should increase certainty regarding target location, and accuracy of retrieval, compared with considering each cue in isolation. Bar height for a given cue in the left panel represents the strength of that cue at each location. Bar height in the right panel represents the choice probability for each location as a result of cue combination. 


\section{Computational formulation of cue combination}

Following standard formulations of cue integration (e.g. Ernst \& Banks, 2002), we model spatial reorientation as probabilistic inference concerning a target location based on a set of independent cues that the target has generated. Specifically, we model the location of the target object $t$ as generating four primary cues $G, A, P, L$ (see illustration in Figure 2), and we model the agent's belief concerning the target location as inference from those cues to $t$.

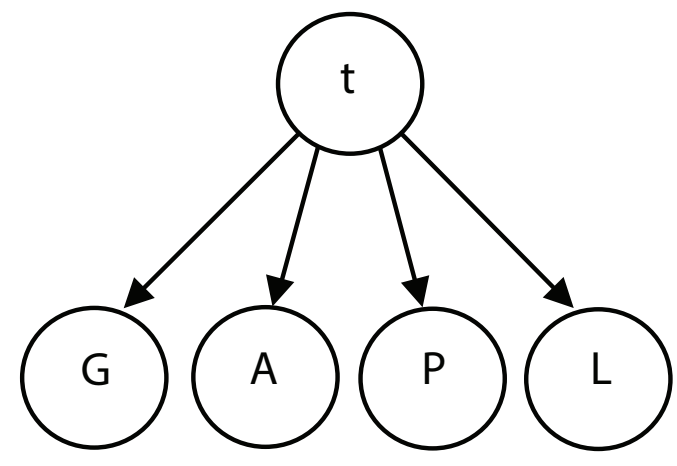

Figure 2: Graphical representation of the combination model. $t$ represents target location. Primary cues are formulated in terms of how they arise from and thus provide information about $t$ : $G$ for geometric cue, $A$ for associative cue, $P$ for polarizing cue, $L$ for language cue. Arrows indicate direction of causation.

More specifically, $G$ (geometry) is informed by the geometric shape of the space, e.g. the lengths of the walls, and the angles they form where they meet. $A$ (direct association) assumes the target is located coincidentally or in the vicinity of a feature-bearing landmark, such as a corner with one white and one colored wall, so that reorientation can be achieved by linking the target directly with the presence of the wall and its location. $P$ (polarization) assumes that the agent has oriented, or polarized, the space using a distinctive feature-bearing landmark, and encodes the location of the target object relative to the landmark. For example, one might locate a coffee shop by remembering how it is situated relative to a bus stop opposite the street, i.e. not directly neighboring it as in the case of the $A$ cue. In rectangular rooms, $A$ and $P$ cannot be disentangled, because each corner is featurally distinctive (i.e., either allwhite or half-colored and half-white) but in rooms with more corners, it is possible to evaluate $A$ and $P$ separately. $L$ (language) is the ability to use spatial language to assist in locating the target. We model language as an independent cue rather than as a tool for bridging across modules because the language-as-bridge approach cannot explain the room size effect, and we wish to determine whether our 
adaptive cue combination view can account for such data.

With the primary cues specified, we cast reorientation as probabilistic inference over possible target locations given the combined set of cues. Using Bayes' rule, we express the posterior belief concerning target location $t$ as:

$$
p(t \mid G, A, P, L) \propto p(G, A, P, L \mid t) p(t)
$$

In a novel environment, we assume a uniform prior $p(t)$ on possible locations reflecting the fact that the participant would have no a priori preference for one location over others. In a situation where there is prior exposure or training in an environment, we can update this posterior distribution by recursively incorporating prior experience or history (discussed below).

The likelihood $p(G, A, P, L \mid t)$ captures the probabilitiy of encountering a specific combination of cues $G, A, P, L$ given that the target is at location $t$. We assume that each cue contributes independently to reorientation, and thus we further decompose the likelihood by separating out the cues using this independence assumption:

$$
p(G, A, P, L \mid t)=p(G \mid t) p(A \mid t) p(P \mid t) p(L \mid t)
$$

It is worth noting that although cues do not interact in the above equation, their contributions are combined in influencing the posterior belief. This formulation for the combination of independent cues is drawn from the literature on perceptual cue integration (Yuille \& Bülthoff, 1996; Ernst \& Banks, 2002; Jacobs, 2002; Knill \& Pouget, 2004). This model setup is also consistent with recent work on reorientation and navigation in ants (Cruse \& Wehner, 2011; Reid, Narendra, Hemmi, \& Zeil, 2011; Collett, 2012; Wystrach, Schwarz, Baniel, \& Cheng, 2013; Legge, Wystrach, Spetch, \& Cheng, 2014).

Each cue can be further decomposed into a likelihood function that specifies how likely it is that the target $t$ will appear in a certain location for that cue, weighted by a prior belief for the same cue.

$$
p(G, A, P, L \mid t) \propto f(t \mid G) p(G) f(t \mid A) p(A) f(t \mid P) p(P) f(t \mid L) p(L)
$$

For all of the analyses, we assume that priors on primary cues are uniform, although our framework makes it possible to encode prior preferences over cues. Thus what remains is to specify the cue likelihood terms $f(t \mid \cdot)$. 
For each cue, we represent its likelihood by a function that indicates how likely the target is to be at each location, given that cue. Because a likelihood function is not a probability distribution, values specified by this function need not sum to 1 . Each cue likelihood function encodes possible target locations predicted by a cue as 1 (illustrated by the tall bars in the left panel of Figure 1) and non-target locations as a noise term $\delta$, capturing general cognitive uncertainty (illustrated by the short bars in Figure 1). For mathematical convenience we add noise only to the non-target locations, thus retaining a maximum value of 1 for the likelihood function; this is formally equivalent to the conceptually natural idea of adding noise to all locations equally, and then rescaling all bars proportionally so that the highest is of height 1 . The noise term $\delta$ is assumed to decrease as a function of age, yielding increasingly targeted, and decreasingly random, choices:

$$
\delta=\frac{1}{w \times a g e}
$$

We use a single weighting parameter $w$ for all cues to specify the inverse relationship between $\delta$ and age: this parameter governs the relative weighting of target and non-target locations. To allow for the simplest design, we also assume that $w$ is the same across cues and is therefore the only free parameter in the model. Thus, this model holds that children are more likely than adults to receive noisy, uncertain information from any given cue, perhaps due to factors such as attention or memory, capturing the qualitative observation that younger children are more likely to make incorrect and somewhat random choices overall concerning target location.

For the $G$ (geometry) cue, the likelihood function assigns a value of 1 for a location $i$ if that cue predicts location $i$ as the target. Often the $G$ cue is not fully reliable since it predicts multiple locations as potential targets, and for this reason, it may be desirable to combine information from multiple cues. ${ }^{1}$ For example, when reorienting in a rectangularly shaped room with a target at one of the four corners, $G$ has a value of 1 on the target corner and on the corner diagonally opposite it, because those are the two locations that are identical to the target location when considering only geometric information. The likelihood also specifies a non-target component, for each location not predicted by this cue, that scales inversely with age subject to a multiplicative weighting parameter as described above.

\footnotetext{
${ }^{1}$ Although the $G$ cue is often unreliable in a controlled experiment with rectilinear spaces, it can be fully predictive in nature.
} 


$$
f(t=i \mid G)= \begin{cases}1 & (\text { if } G \text { predicts } i) \\ \delta & \text { (otherwise) }\end{cases}
$$

The likelihood of the $A$ (associative) cue has a value of 1 on locations where a feature-bearing landmark coincides with the target. For example, when reorienting in a rectangularly shaped room with one of the four walls providing a feature or landmark (e.g. painted blue), $A$ has a value of 1 on each of two corners, because the landmark is ambiguous about which of the two all-white corners, or which of the two half-blue corners, is the correct target if only direct associative strategies are used. In addition, the strength of the $A$ cue depends on the salience of the landmark (e.g. the salience of the colored wall). This component would be perfectly informative in a high-salience condition, but uninformative in a low-salience condition. This dependence on salience is motivated by previous findings that a small enclosure can degrade reorientation performance despite the presence of a landmark feature, especially in very young children (Hermer \& Spelke, 1996). ${ }^{2}$ We assume that small room size reduces the salience of the landmark feature, a choice we will discuss further after presenting the data and modeling. We formalize this interaction between salience and the $A$ cue using a probabilistic OR.

$$
f(t=i \mid A)= \begin{cases}1-(1-s)(1-\delta) & \text { (if } A \text { predicts } i) \\ \delta & \text { (otherwise) }\end{cases}
$$

Here $s$ represents salience and can range from 0 (no salience) to 1 (full salience). When $s$ is 0 , this likelihood function is entirely flat and uninformative. Here, the salience parameter $\mathrm{s}$ is assumed to be 0 in a small room (area no greater than 24 square feet, motivated by previous work by Hermer \& Spelke, 1996), and 1 otherwise. This binary assumption is made for simplicity, but future work should systematically explore independent empirical measures of landmark salience for rooms of different sizes. The parameter $w$ for the $\delta$ term here and for other cues below is the same as for the $G$ cue.

The likelihood of the $P$ (polarizing) cue assigns 1 on the true target location under the assumption that the landmark object can polarize the space and orient the participant towards one of the corners.

\footnotetext{
${ }^{2}$ Although it is possible that other cues are also affected by salience, here we choose to model the effect of salience on the $A$ cue only based on empirical evidence from studies on the room-size effect.
} 


$$
f(t=i \mid P)= \begin{cases}1 & (\text { if } P \text { predicts } i) \\ \delta & \text { (otherwise })\end{cases}
$$

The likelihood of the $L$ (language) cue depends on the availability of spatial language:

$$
f(t=i \mid L)= \begin{cases}1-(1-a(L))(1-\delta) & (\text { if } L \text { predicts } i) \\ \delta & \text { (otherwise) }\end{cases}
$$

This cue points to the target and becomes available when an agent is fluent and has access to relevant spatial language, which is determined by the function $a(L)$. This function ranges from 0 (no access to spatial language) to 1 (full access). We assume a value of 0 (no access) for participants under the age of 6 , a value of 1 (full access) for those who are 6 years of age or older and who are not experimentally prevented from using language (e.g., by a concurrent verbal interference task), and a range of values between 0 and 1 for participants 6 years of age or older who are being experimentally prevented from

using language. These assumptions are motivated by previous work suggesting that spatial language helps children of age 6 but not younger in reorientation (Hermer-Vazquez, 1997; Hermer-Vazquez, Moffet, \& Munkholm, 2001) and by work on auditory shadowing (Hermer-Vazquez, Spelke, \& Katsnelson, 1999). In our studies below, we test the hypothesis that the assumptions we have made here, together with general principles of probabilistic inference, account for human reorientation behavior across a range of published experiments.

\section{Data}

To assess our model, we used empirical data from several existing published sources. We chose these data sets to cover the breadth of phenomena reported in the reorientation literature while maintaining analytical consistency across studies; for example, we restricted our analysis to experimental conditions where the landmark is close to a wall. Table 2 summarizes the phenomena and empirical data relevant to our analyses and their theoretical implications. 


\begin{tabular}{c|l|l|l} 
Case & Phenomenon & Theoretical implication & Source \\
\hline \hline 1 & $<6 y r$ fail to use landmark in small enclosure & Enclosure size matters & LNN02; HS96 \\
\hline 2 & $<6 y r s$ use distant landmark to reorient & Ppatial language helps reorientation & RN08a; HS96 \\
\hline 3 & Adults trained in large enclosure favor landmark & Experience and enclosure size interact & RN08b
\end{tabular}

Table 2: Summary of data sets used in our three case studies. LNN02: Learmonth, Nadel \& Newcombe (2002); HS96: Hermer \& Spelke (1996); RN08a: Ratliff \& Newcombe (2008a); NRST10: Newcombe, Ratliff, Shallcross \& Twyman (2010); RN08b: Ratliff \& Newcombe (2008b).

\section{Model evaluation and results}

We evaluate our model in three independent test cases. First, we examine how well our model accounts for data in reorientation tasks in a rectangular room, when association and polarization cues cannot be distinguished because targets are always spatially coincident with a feature-bearing landmark. We compare our combination model both to the associative learning model for human reorientation (Miller, 2009) and to baseline single-cue models that do not use cue combination. We begin in case study 1 by fitting our model to data from children in a standard reorientation task, thereby obtaining an estimate of the one model parameter, which governs the rate at which uncertainty decreases with age. To assess the generalizability of our model in a predictive way, we then apply our model to predict data from adults in a similar experiment, and subsequently to data from an independent set of tasks involving verbal interference, polarizing cues, and cue conflict under prior experience, in case studies 2 and 3, all using the fitted parameter value from the first step.

\section{Case study 1: Cue combination in a rectangular enclosure}

We first assess whether our proposed combination model can account for data from rectangular rooms. We draw on a representative data set that spans several age groups (Learmonth et al., 2002). This set was used by Miller (2009), so we can directly compare our model results to Miller's.

The experimental procedures are described fully in Learmonth et al. (2002); we describe them briefly here. All experiments were carried out in an enclosed rectangular room. The size of the room differed 
across two conditions: large $(8 \times 12 \mathrm{ft})$ and small $(4 \times 6 \mathrm{ft})$. In both cases, one of the shorter walls had a blue curtain that served as a landmark (the "blue-wall condition"). Figure 3a illustrates this experimental setup. The target was placed at one of the four corners for a given participant. The four corners of the room were referred to as the $C$ (correct), $R$ (rotationally identical or invariant), $N$ (near) and $F$ (far) corners. Three groups of children from 3 to 6 years of age participated in the reorientation task. In each trial, participants were shown the target location, disoriented and then asked to point to the target. Figure 3b-c shows the average empirical choice probabilities, along with model fits as described below.

We modeled these data using our cue combination model as follows. The $G$ cue was encoded by a likelihood function that includes $C$ and $R$ corners as possible targets. This captures the fact that the $C$ and $R$ corners are geometrically identical or rotationally invariant (Hermer \& Spelke, 1996); thus geometry ambiguously predicts target location. The $A$ cue encodes $C$ and $N$ as possible targets. This captures the fact that the target and its nearest corner coincide either with the landmark blue wall, or not. However, the likelihood of the $A$ cue is dependent on salience, which in this case is assumed to be determined by room size. We make the simplifying assumption that in the small room, salience $s=0$ ; in the large room, salience $s=1$. The $P$ cue is not present (i.e. its likelihood is uniform) because the landmark coincides with the target. For the $L$ cue, we assumed full access to spatial language $(L(a)=1)$ for participants 6 years of age or older who were not under verbal interference, no access to spatial language $(L(a)=0)$ for participants under 6 years of age, and an intermediate value of $L(a)$ for participants 6 years or older who were under verbal interference. The only free parameter in the model is $w$, which determines how quickly general cognitive uncertainty decreases as a function of age. We fit this parameter to the data through grid search by incrementally varying the value between 0.01 and 1 in steps of 0.01. Specifically, we iteratively generated model-estimated choice probabilities for each value of the parameter. We then identified the parameter value that yielded the minimal mean squared error between model-estimated choice probabilities and the empirical data. We found the optimal parameter value to be 0.694 and held that parameter fixed in the current analysis and in all upcoming predictive analyses.

We compared the choice probabilities estimated from our combination model to those from Miller's (2009) associative model (see model specification in Supplementary Materials), as well as a baseline model that involves only the $G$ cue and a second baseline model that involves only the $A$ cue, with the same likelihood specifications as in the combination model but neither enabling cue combination. Figure 3b-c 
summarizes the results. In the large-room condition (Figure 3b), performance is almost indistinguishable between the combination $\left(r^{2}=0.98\right)$ and Miller's $\left(r^{2}=0.97\right)$ models, and both outperformed the baseline models $\left(r^{2}=0.41\right.$ for $G$-cue model and $r^{2}=0.30$ for the $A$-cue model). This result is expected because in the large room, the language cue $L$ (which is absent from Miller's model) is redundant given jointly the associative cue $A$ and geometrical cue $G$ (both of which are present in Miller's model as associative cues), since both cues correctly point to the target in combination; thus both of these models have this critical information, and the baseline models lack them because neither geometry nor direction alone is sufficient to pick out the correct target. In the small-room condition (Figure 3c), both Miller's $\left(r^{2}=0.73\right)$ and two baseline $\left(r^{2}<0.55\right)$ models perform poorly in explaining the data, but our proposed model accounts for the choice pattern substantially better $\left(r^{2}=0.97\right)$. In particular, Miller's model predicts higher choice probability on the correct corner than other corners for all age groups, and hence it fails to predict the qualitative difference in performance between age groups $<6$ and $=6$. On the other hand, both the $G$-cue model and the $A$-cue model fail to capture the superior performance in age group 6 altogether. This is because neither of these models captures the presumed influence of language when salience is weak in the small room. Our model, however, does explicitly capture this interaction between language and salience, and best explains these empirical findings that contrast reorientation performance across ages between large and small enclosures. 


\section{a)}
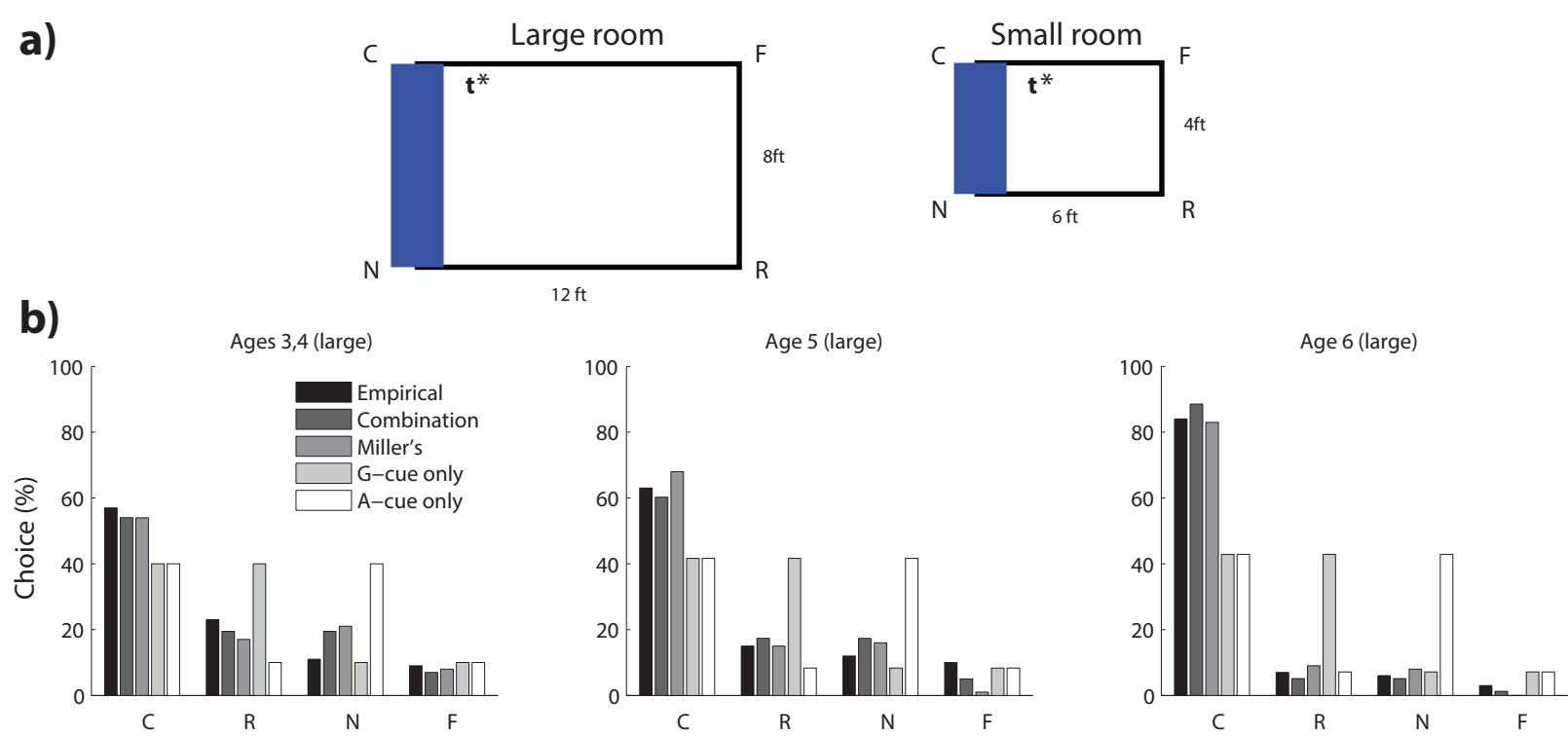

c)
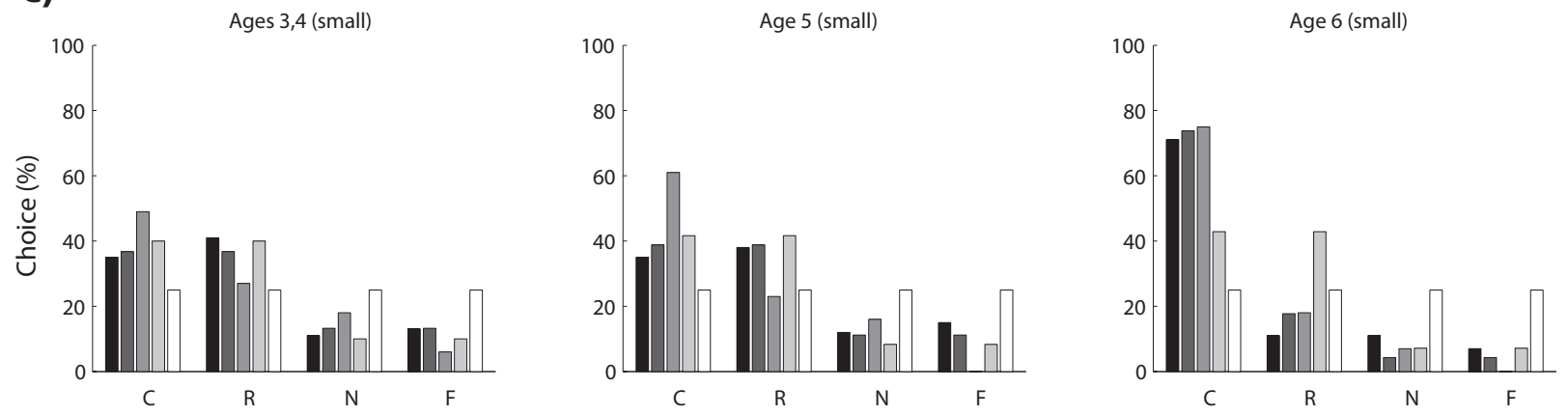

Figure 3: Summary of analyses on cue combination in children. a) Large and small rectangular rooms (not-to-scale) used in the experiments by Learmonth et al. (2002). t* marks target location. b) Empirical (standard errors were not provided in Learmonth et al. (2002) and thus are not shown here) and modelestimated choice probabilities from children for the large room. c) Similar choice probabilities for the small room

To further assess our model, we used the parameters estimated from the previous experiment to predict data from a separate study conducted with adults (Ratliff \& Newcombe, 2008a). We took adults' age to be 18 and used the same parameter value for $w$ from the previous analysis. The specific paradigm we tested is consistent with the blue-wall experiment described earlier: adults were disoriented in identically sized large and small rooms. However in this study, one of the conditions involved verbal shadowing, intended to render language partially inaccessible during reorientation (see also Hermer-Vazquez et al., 1999). Figure 4 (first column) presents data from Ratliff \& Newcombe (2008a). It can be seen that 
verbal shadowing appears to somewhat reduce the rate of correct responding, and that this reduction is (significantly) smaller in the large room than in the small room. We interpret the greater resilience of correct responding under verbal shadowing in the large room as resulting from the greater salience of the landmark in the large room (supporting the $A$ cue), despite the experimentally induced degradation of the $L$ (language) cue in both conditions. Figure 4 (columns 2 onwards) presents the response patterns predicted by the same three models described above. To obtain these results, we varied the availability of language $a(L)$ in the $L$ cue over the range 0 to 1 (in steps of 0.1 ) to capture different degrees of language inaccessibility, effectively marginalizing out the possible values that $a(L)$ can take; the bar graphs for the combination model in the shadowed condition show the average prediction within that range. In contrast bar graphs in the No-shadow condition were obtained by assuming full access to spatial language $(a(L)=1)$. Our model again outperforms $\left(r^{2}=0.98\right)$ all the competing models $\left(r^{2}<0.1\right)$ by capturing the interaction between salience (here, room size) and language, whereas the competing models do not account for this interaction.
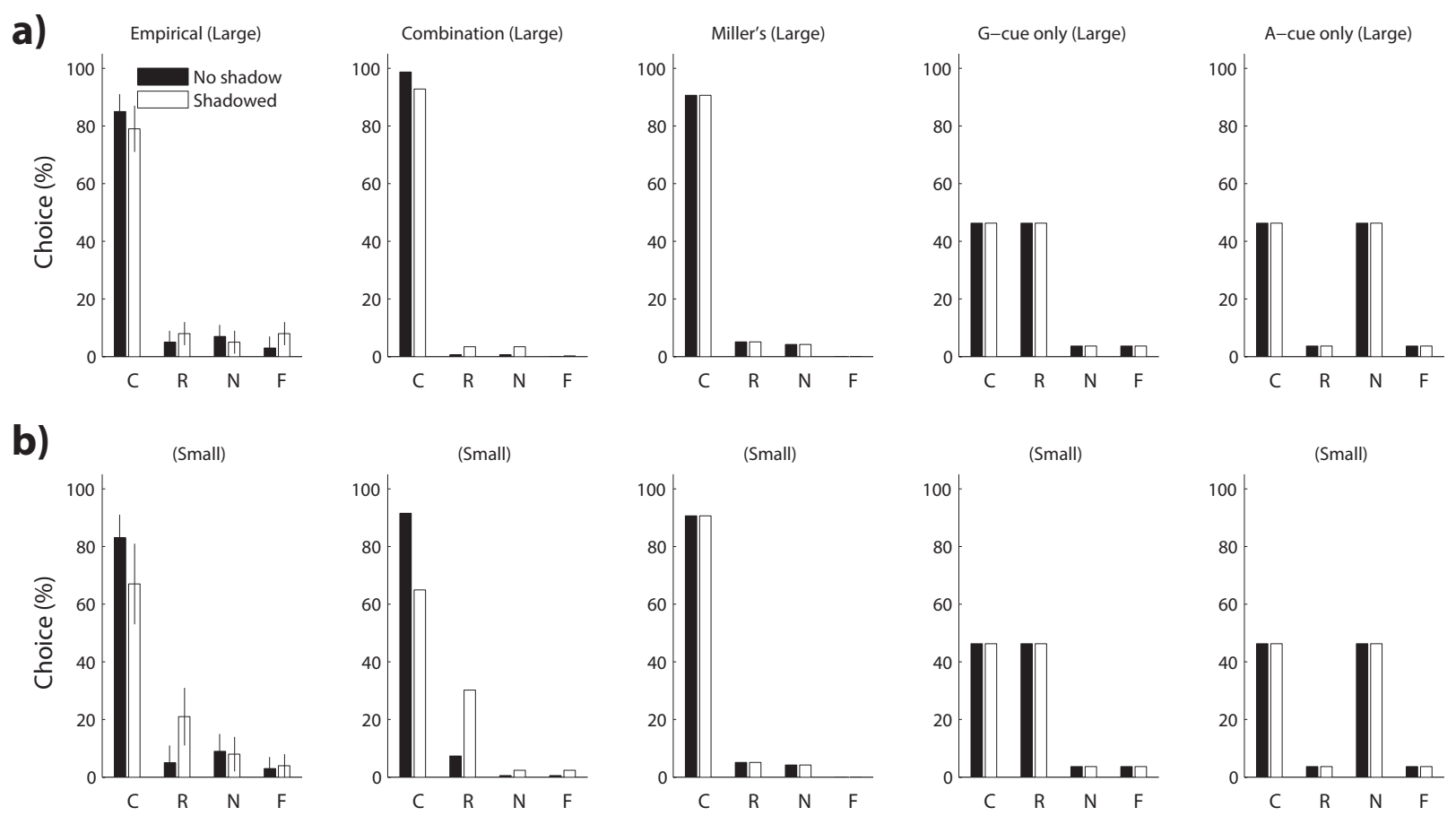

Figure 4: Summary of analyses on cue combination in adults. a) Empirical and model-estimated choice probabilities under control and verbal-shadowing conditions for the large room. Thin vertical lines represent standard errors from Ratliff \& Newcombe (2008a). b) Analogous choice probabilities for the small room. 
To assess the model performances, we also compared the log likelihood of model fits to the empirical data. We used log likelihood instead of Akaike information criterion or Bayesian information criterion because our model used fewer parameters than the associative model in general (and it used an equal number of parameters with respect to other baseline models), and hence it has lower complexity. Table 3 compares both log likelihoods and number of free parameters among the different models - our model yields better performances while having minimal complexity under all conditions in this case. Taken together, these two sets of results suggest that our cue combination model accounts well for these reorientation data.

\begin{tabular}{|c|c|c|c|c|c|}
\hline Case & Condition (group) & \multicolumn{3}{|c|}{ Log likelihood (\# distinct params) } & \\
\hline \multirow[t]{4}{*}{1} & & Combination & Miller's & G-cue & A-cue \\
\hline & Large rectangle (children) & $-5.26(1)$ & $-5.66(4)$ & $-8.47(1)$ & $-8.66(1)$ \\
\hline & Small rectangle (children) & $-5.09(1)$ & $-7.47(4)$ & $-7.59(1)$ & $-8.31(1)$ \\
\hline & Shadowing (adult) & $-6.69(1)$ & $-10.57(4)$ & $-10.04(1)$ & $-10.04(1)$ \\
\hline \multirow[t]{2}{*}{2} & & Combination & Miller's & G-cue & P-cue \\
\hline & Polarizing (children) & $-5.22(1)$ & $-6.57(4)$ & $-6.88(1)$ & $-7.29(1)$ \\
\hline \multirow[t]{2}{*}{3} & & Combination & Combination (flat prior) & G-cue & A-cue \\
\hline & Experience-based (adult) & $-6.87(1)$ & $-8.75(1)$ & $-10.17(1)$ & $-7.58(1)$ \\
\hline
\end{tabular}

Table 3: Summary of model performances across three case studies. Best score in each row is shown in bold.

\section{Case study 2: Cue combination in an octagonal enclosure}

We next assess whether our model also accounts for cue combination beyond a rectangular enclosure. In particular, we focus on polarizing cue combination in an octagonal enclosure, as reported by Newcombe et al. (2010).

In this set of experiments, the reorientation task was conducted in an octagonal room. Similar to the previous experiments, one of the walls had a red curtain that served as a landmark. However in contrast with the previous experiments, the target location did not coincide with any of the corners covered by the landmark, but instead was one of the distant corners as illustrated in Figure 5a (leftmost 
panel). In other words, successful reorientation in this case would depend on mapping from a distant landmark to a target, or direction. The room was 75 square feet in area, which is close to the size of the previous large rectangular room. Two of the corners $(G W)$ were geometrically identical to the correct corner $(C)$. One corner $(G F)$ was both geometrically identical and coincides with the featured landmark. The remaining three corners were error corners $(E W)$. Two groups of children (ages 3 and 5 ) participated in the experiment. Figure $5 \mathrm{~b}$ (right 2 panels) shows the empirical choice probabilities, and model predictions of them.

We used the combination model to predict these empirical data. Specifically, the $G$ cue encodes geometrically invariant corners $(C, G F, G W$ and $G W)$ as possible targets and non-targets elsewhere. The polarizing $P$ cue encodes $C$ as the possible target. The $L$ cue is uninformative in this case because both age groups for the octagonal experiments are below 6, which we assume means that language is unavailable, yielding a flat likelihood for the $L$ cue. To allow for a fully predictive (parameter-free) assessment, we used the $w$ values for 3 - and 5-year-olds from the blue-wall experiments (our study 1), which are independent of the current analysis. ${ }^{3}$

We compared our combination model against two baseline models that incorporate either the polarizing cue alone, or the geometrical cue alone, with no cue combination. Figure 5b summarizes the predicted choice probabilities from all three models. These results show that our combination model $\left(r^{2}=0.90\right)$ predict empirical data across two age groups better than Miller's model $\left(r^{2}=0.80\right)$ and both the $G$-cue $\left(r^{2}=0.35\right)$ and $P$-cue $\left(r^{2}=0.84\right)$ baseline models. The $P$-cue only model cannot distinguish between geometrically identical corners and error corners, so it predicts equal choice probabilites over those corners. The geometry-only model cannot distinguish between the correct corner and geometrically equivalent corners, so it under-predicts choices on the target. Table 3 further shows that our model yields better log likelihood than competing models. In sum, this set of results suggests that children combine directional information with geometry in spatial reorientation, which extends beyond an associative account and provides further evidence for a cue-combination view.

\footnotetext{
${ }^{3}$ We assumed $w$ to be fixed across experiments to support a fully predictive analysis. However, we confirmed that there exist $w$ values that give better fits to the current study, suggesting $w$ might be different under rectangular and octagonal conditions.
} 

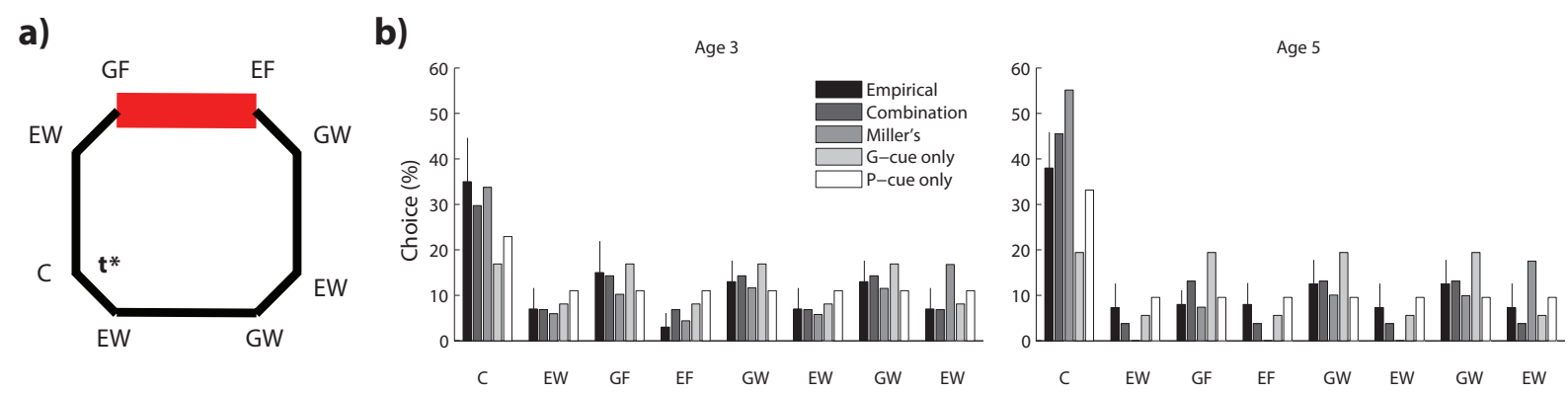

Figure 5: Summary of analyses on directional cue combination. a) Octagonal room used in the experiments by Newcombe et al. (2010). b) Empirical and model-predicted choice probabilities for 3- and 5-year-olds. Thin vertical lines represent standard errors.

\section{Case study 3: Cue combination from experience}

Finally, we assess our model in a reorientation experiment that involves prior experience. We focus on a cue-conflict paradigm based on experimental data reported by Ratliff \& Newcombe (2008b).

In these experiments, the reorientation tasks were conducted in rectangular rooms with sizes (small or large) identical to those in Learmonth et al. (2002). Differing from the previous experiments, however, these tasks involved a training phase and a separate testing phase. During training, the experimenter would hide a target object in one of the four corners. Meanwhile, a landmark (a removable piece of bright colored and patterned fabric) would be placed close to that target location. Throughout training, the target would be hidden in the same corner, and participants would be disoriented and asked where the target was hidden. During testing, the participants would be taken out of the room while the experimenter went back in and switched the landmark to the adjacent wall. Participants were then blindfolded and led back into the room for the test. After the disorientation procedure, participants were asked to point to the corner where they thought the target object was located. Figure 6a illustrates this paradigm. Note that due to the shift of the landmark, the geometry and landmark cues would be in conflict with each other during the test. In particular, geometry would point to two $G$ (see right panel in Figure 6a) corners as potential target locations, whereas landmark would point to $L$; the remaining corner is labeled $E$ (error). The experiments involved two conditions. In the same-room condition, the training and testing rooms were of the same size. In the switched-room condition, the training and testing rooms were of different sizes (either small or large). Adults of age around 18 participated in the 
study. Figures $6 \mathrm{~b}$ and $6 \mathrm{c}$ show the empirical choice probabilities, and model predictions of them.

We used our combination model to predict these data as follows. To incorporate the training experience in the inference of target locations, we used recursive posterior updating. Specifically, Equation 1 can take into account history, or training experience:

$$
p(t \mid c u e s) \propto p(\text { cues } \mid t) p\left(t \mid \text { cues }^{\prime}\right)
$$

Here, $p($ cues $\mid t)$ is the likelihood that specifies cues in the testing phase. cues $^{\prime}$ refer to cues in the training phase, and the prior for the testing phase $p\left(t \mid c u e s^{\prime}\right)$ is obtained from the posterior in the training phase:

$$
p\left(t \mid \text { cues }^{\prime}\right) \propto p\left(\text { cues }^{\prime} \mid t\right) p(t)
$$

$p(t)$ is the prior in the training phase that is assumed to be uniform, i.e. there is no preference towards any location before the training.

The model thus makes predictions about target choice during testing. We specified the likelihoods for the $G, A$, and $L$ cues according to Equations 4-7: $G$ cue encodes the two $G$ corners as possible targets; $A$ cue encodes the $L$ corner as the target (i.e. in conflict with $G$ cue) in the large room and is uninformative (flat) in the small room; $L$ cue encodes the $L$ corner as the target; $P$ cue has a uniform likelihood (as in study 1). For the same-room condition, many participants noticed that the landmark had shifted from training to testing (as reported in Ratliff \& Newcombe (2008b)). For this reason, they were likely to consider the landmark an unreliable feature, although the exact degree of reliability of this cue might vary across participants. To capture this observation, we took into account varying degrees of uncertainty in the likelihood function by marginalizing out the $w$ parameter such that the non-targetto-target ratio covers the range from 1:1 (where a cue is completely flat or uninformative) to $1: 10$ (the lowest target-to-non-target ratio as predicted by the model informed by the empirical data in study 1) in incremental steps of 1 in landmark-relevant cues $A$ and $L$. We then took the average model prediction within this range, effectively marginalizing out the free parameter.

We compared the choice probabilities predicted from our combination model to models that do not incorporate cue combination. We did not include the associative learning model because its original formulation did not specify how to incorporate rapid history effects in this context. Figure $6 \mathrm{~b}$ and $6 \mathrm{c}$ 
summarize these results. Overall, the combination model makes accurate predictions about the empirical data $\left(r^{2}=0.93\right)$. It captures the phenomenon that exposure to the large room (regardless of whether during training or testing) leads to a preference towards the landmark corner $L$, whereas an exclusive exposure to the small room reduces the choice probability at $L$ and increases the probabilities at the geometric corners $G$. The combination model predicts these data better than competing models that incorporate only the associative $A$ cue $\left(r^{2}=0.86\right)$ or geometry $G$ cue $\left(r^{2}=0.09\right)$ across all conditions, again supporting the cue-combination view. Finally, to verify that prior experience did play an important role in model prediction, we ran a version of the combination model with a uniform prior on target locations in the testing phase (not shown in the figure). This yielded a performance $\left(r^{2}=0.53\right)$ that is substantially worse than the version with prior incorporated. Table 3 shows that our model outperforms all competing models.

Together, this set of results supports the cue-combination view and points to an interaction of enclosure size and reorientation history, both of which serve as important factors in the strategic choice of target. 
a)

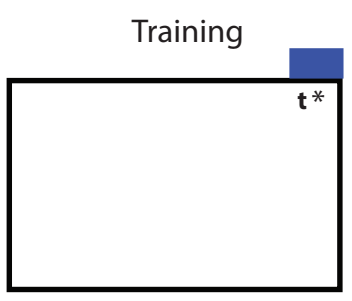

b)

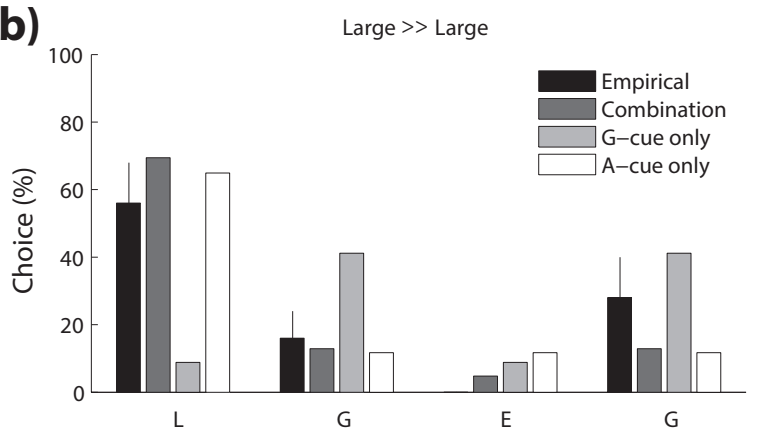

c)

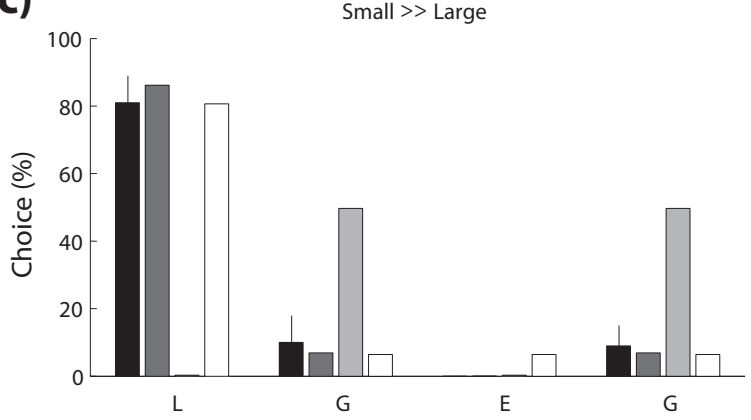

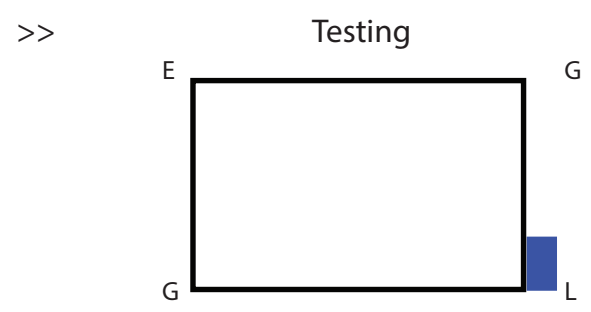
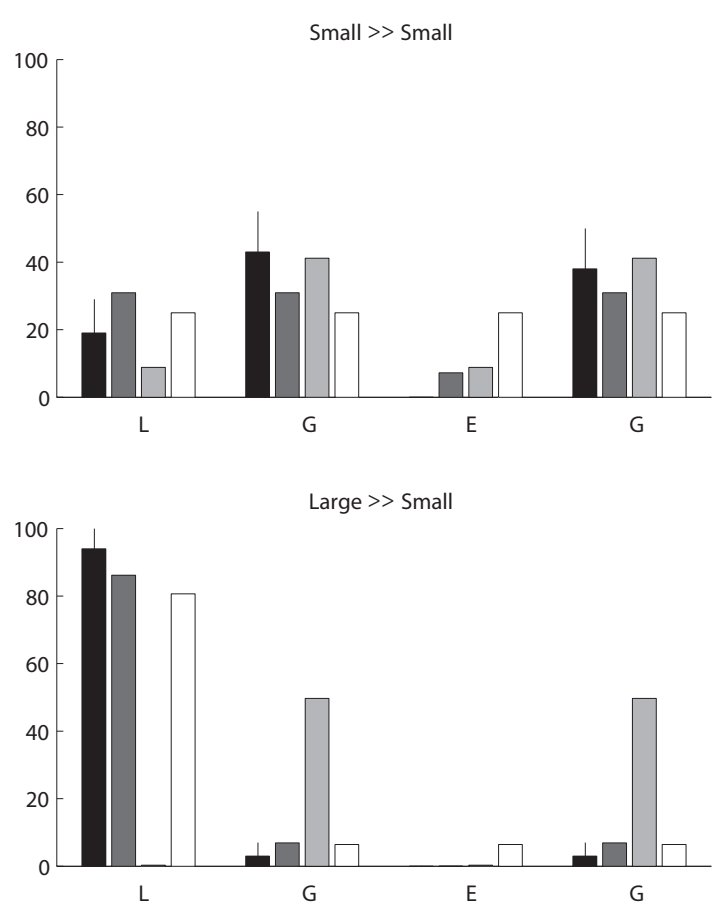

Figure 6: Summary of analyses on cue combination that incorporates experience. a) Training and testing procedures of the cue-conflict paradigm in Ratliff Newcombe (2008b). b) Empirical and model-predcited choice probabilities in the same-room condition. c) Choice probabilities in the switched-room condition. Thin vertical lines represent standard errors.

\section{Discussion}

We have presented a probabilistic model of the development of human spatial reorientation based on cue combination. Our model accounts for existing data better than modularity or associative models, across different enclosure sizes and shapes, different age groups, different task demands, and different landmark configurations. Several aspects of this model deserve discussion.

First, we treat language as an independent cue, not as a tool for combining information from other 
cues. This treatment differs from the modular approach. Our treatment of language as a cue among others, rather than as a tool for combining information from other cues, receives some independent support from the finding of Shusterman, Lee and Spelke (2011) that non-specific task-relevant language can help young children in the reorientation paradigm (e.g., the red wall can help you find the sticker), and from the finding of Dessalegn and Landau (2013) that any asymmetrical language aids in capturing directional spatial relations (e.g., the red is prettier than the blue, as well as the red is left of the blue).

Second, we did not explicitly model learning in children, because such effects have been found to be minimal in the developmental literature. However, we did demonstrate that our model is capable of capturing prior experience in adults (in case study 3), which is a particular form of learning that could be qualitatively different from those observed in other non-human species - where repeated trials are essential to successful spatial reorientation. It remains an open question why children show minimal learning in existing spatial reorientation experiments, and how they might behave in experiments where more trials are given or when cues are placed in conflict, as in the case study with adults.

Third, we should note that the current model treats separately changes due to age (modeled by decreasing cognitive uncertainty with age) and changes due to short-term experience (modeled by recursive updating). An open question for future research is whether decreases in uncertainty with age may also be attributable to experience, accumulated over developmental time rather than over the short run, yielding a simpler overall account. An alternative is that age-related decreases in uncertainty are due to more general aspects of cognitive development, such as better executive control of attention and retrieval. Another aspect of the model that could be adjusted involves the capacity to combine information, which may also change with age,e.g., factors such as hippocampal maturation could act to allow increasing ease in combining information from various cues as children get older (Sutton \& Newcombe, 2014). Indeed, developmental research regarding combination in other kinds of spatial problems has shown an extended period of development (Nardini, Bales, \& Mareschal, 2015; Nardini, Burgess, Breckenridge, \& Atkinson, 2006; Waismeyer \& Jacobs, 2013).

Finally, we modeled the room-size effect by varying the salience of the featural cue rather than the salience of the geometric cue. Previous discussion of the room size effect has included suggestions that geometric cues may be more noticeable in smaller rooms (Twyman, Nardi, \& Newcombe, 2013). However, an approach that varied the salience of geometry would have difficulty in accounting for the continued dominance of the reversal error over the near and far errors in larger rooms where the frequency of the 
correct choice increases sharply.

Our model was developed to account for human data, and further work would be needed to extend it to evaluate data from non-human species, and from other paradigms with humans. In contrast, the associative model (Miller \& Shettleworth, 2007) was originally developed to account for findings from non-human animals who require lengthy learning periods in typical experimental paradigms, and was later extended to account for human data (Miller, 2009). Clearly, a model for non-human species would not use our independent language factor, and there may be other species-specific aspects to reorientation, as suggested by Cheng, Huttenlocher, \& Newcombe (2013). Further work is needed to determine which of the principles we have explored here generalize across species, and how.

The work we have presented here has helped to account for empirical findings in human spatial reorientation in children and adults - often considered puzzling - under the principle of adaptive cue combination, a proposal that has not previously been computationally specified or tested with respect to these phenomena. The model is grounded in the extensive literature on perceptual cue integration and memory for spatial location, thus suggesting that human spatial reorientation may be an instantiation of optimal behavior under uncertainty.

\section{Acknowledgments}

Work on this project was funded by a grant to the Spatial Intelligence and Learning Center from the National Science Foundation, SBE 1041707.

\section{References}

Berniker, M., \& Kording, K. (2011). Bayesian approaches to sensory integration for motor control. Wiley Interdisciplinary Reviews: Cognitive Science, 2(4), 419-428.

Brown, A. A., Spetch, M. L., \& Hurd, P. L. (2007). Growing in circles rearing environment alters spatial navigation in fish. Psychological Science, 18, 569-573.

Cheng, K. (2008). Whither geometry? Troubles of the geometric module. Trends in Cognitive Sciences, 12, 355-361. 
Cheng, K., Huttenlocher, J., \& Newcombe, N. S. (2013). 25 years of research on the use of geometry in spatial reorientation: A current theoretical perspective. Psychonomic bulletin Es review, 20, 1033-1054.

Cheng, K., Shettleworth, S. J., Huttenlocher, J., \& Rieser, J. J. (2007). Bayesian integration of spatial information. Psychological Bulletin, 133, 625-637.

Chiandetti, C., Regolin, L., Sovrano, V. A., \& Vallortigara, G. (2007). Spatial reorientation: The effects of space size on the encoding of landmark and geometry information. Animal Cognition, 10, 159-168.

Chiandetti, C., \& Vallortigara, G. (2008). Is there an innate geometric module? Effects of experience with angular geometric cues on spatial re-orientation based on the shape of the environment. Animal Cognition, 11, 139-146.

Chiandetti, C., \& Vallortigara, G. (2010). Experience and geometry: Controlled-rearing studies with chicks. Animal Cognition, 13, 463-470.

Collett, M. (2012). How navigational guidance systems are combined in a desert ant. Current Biology, 22, 927-932.

Cruse, H., \& Wehner, R. (2011). No need for a cognitive map: Decentralized memory for insect navigation. PLoS Computational Biology, 7, e1002009.

Dessalegn, B., \& Landau, B. (2013). Interaction between language and vision: Its momentary, abstract, and it develops. Cognition, 127, 331-344.

Ernst, M. O., \& Banks, M. S. (2002). Humans integrate visual and haptic information in a statistically optimal fashion. Nature, 415, 429-433.

Gallistel, C. R. (1990). The organization of learning. The MIT Press.

Hermer, L., \& Spelke, E. (1996). Modularity and development: The case of spatial reorientation. Cognition, 61, 195-232.

Hermer, L., \& Spelke, E. S. (1994). A geometric process for spatial reorientation in young children. Nature, 370, 57-59.

Hermer-Vazquez, L. (1997). Cognitive flexibility as it emerges over development and evolution: 
The case of two navigational tasks in humans (Unpublished doctoral dissertation). Cornell University.

Hermer-Vazquez, L., Moffet, A., \& Munkholm, P. (2001). Language, space, and the development of cognitive flexibility in humans: The case of two spatial memory tasks. Cognition, 79, 263-299.

Hermer-Vazquez, L., Spelke, E. S., \& Katsnelson, A. S. (1999). Sources of flexibility in human cognition: Dual-task studies of space and language. Cognitive Psychology, 39, 3-36.

Huttenlocher, J., Hedges, L. V., \& Duncan, S. (1991). Categories and particulars: Prototype effects in estimating spatial location. Psychological Review, 98, 352.

Jacobs, R. A. (2002). What determines visual cue reliability? Trends in Cognitive Sciences, $6(8), 345-350$.

Kelly, D. M., \& Spetch, M. L. (2004). Reorientation in a two-dimensional environment: II. Do pigeons (columba livia) encode the featural and geometric properties of a two-dimensional schematic of a room? Journal of Comparative Psychology, 118, 384-395.

Kelly, D. M., Spetch, M. L., \& Heth, C. D. (1998). Pigeons'(columba livia) encoding of geometric and featural properties of a spatial environment. Journal of Comparative Psychology, 112, 259-269.

Knill, D. C., \& Pouget, A. (2004). The Bayesian brain: The role of uncertainty in neural coding and computation. Trends in Neurosciences, 27(12), 712-719.

Learmonth, A. E., Nadel, L., \& Newcombe, N. S. (2002). Children's use of landmarks: Implications for modularity theory. Psychological Science, 13, 337-341.

Learmonth, A. E., Newcombe, N. S., \& Huttenlocher, J. (2001). Toddlers' use of metric information and landmarks to reorient. Journal of Experimental Child Psychology, 80, 225-244.

Legge, E. L. G., Wystrach, A., Spetch, M. L., \& Cheng, K. (2014). Combining sky and earth: Desert ants (melophorus bagoti) show weighted integration of celestial and terrestrial cues. Journal of Experimental Biology, 217, 4159-4166.

Miller, N. Y. (2009). Modeling the effects of enclosure size on geometry learning. Behavioural 
Processes, 80, 306-313.

Miller, N. Y., \& Shettleworth, S. J. (2007). Learning about environmental geometry: An associative model. Journal of Experimental Psychology: Animal Behavior Processes, 33, $191-212$.

Miller, N. Y., \& Shettleworth, S. J. (2008). An associative model of geometry learning: A modified choice rule. Journal of Experimental Psychology: Animal Behavior Processes, 34 , $419-422$.

Nardini, M., Bales, J., \& Mareschal, D. (2015). Integration of audio-visual information for spatial decisions in children and adults. Developmental Science, 19.

Nardini, M., Burgess, N., Breckenridge, K., \& Atkinson, J. (2006). Differential developmental trajectories for egocentric, environmental and intrinsic frames of reference in spatial memory. Cognition, 101, 153-172.

Newcombe, N. S., \& Huttenlocher, J. (2006). Development of spatial cognition. In D. Kuhn \& R. S. Siegler (Eds.), Handbook of child psychology (6th edition) (pp. 734-776). John Wiley.

Newcombe, N. S., Ratliff, K. R., Shallcross, W. L., \& Twyman, A. D. (2010). Young children's use of features to reorient is more than just associative: Further evidence against a modular view of spatial processing. Developmental Science, 13, 213-220.

Ratliff, K. R., \& Newcombe, N. S. (2008a). Is language necessary for human spatial reorientation? reconsidering evidence from dual task paradigms. Cognitive Psychology, 56, 142-163.

Ratliff, K. R., \& Newcombe, N. S. (2008b). Reorienting when cues conflict evidence for an adaptive-combination view. Psychological Science, 19, 1301-1307.

Reid, S. F., Narendra, A., Hemmi, J. M., \& Zeil, J. (2011). Polarised skylight and the landmark panorama provide night-active bull ants with compass information during route following. Journal of Experimental Biology, 214, 363-370.

Rescorla, R. A., \& Wagner, A. R. (1972). A theory of Pavlovian conditioning: Variations in the effectiveness of reinforcement and nonreinforcement. In A. H. Black \& P. W. F (Eds.), Classical conditioning II: Current research and theory (pp. 64-99). Appleton-Century- 
Crofts.

Shusterman, A., Lee, S. A., \& Spelke, E. S. (2011). Cognitive effects of language on human navigation. Cognition, 120, 186-201.

Sjolund, L., Kelly, J., \& McNamara, T. (2014). Cue integration during navigation. Poster presented at the 55th Annual Meeting of the Psychonomic Society.

Sovrano, V. A., Bisazza, A., \& Vallortigara, G. (2007). How fish do geometry in large and in small spaces. Animal Cognition, 10, 47-54.

Sovrano, V. A., \& Vallortigara, G. (2006). Dissecting the geometric module a sense linkage for metric and landmark information in animals' spatial reorientation. Psychological Science, $17,616-621$.

Sutton, J. E., \& Newcombe, N. S. (2014). The hippocampus is not a geometric module: Processing environment geometry during reorientation. Frontiers in Human Neuroscience, 8, 596.

Twyman, A. D., Friedman, A., \& Spetch, M. L. (2007). Penetrating the geometric module: Catalyzing children's use of landmarks. Developmental Psychology, 43, 1523-1530.

Twyman, A. D., Nardi, D., \& Newcombe, N. S. (2013). Two fields are better than one: Developmental and cognitive perspectives on underatnding spatial reorientation. Comparative Cognition 83 Behavior Reviews, 8, 78-97.

Twyman, A. D., Newcombe, N. S., \& Gould, T. J. (2013). Malleability in the development of spatial reorientation. Developmental Psychobiology, 55, 243-255.

Vallortigara, G., Feruglio, M., \& Sovrano, V. A. (2005). Reorientation by geometric and landmark information in environments of different size. Developmental Science, 8, 393-401.

Waisman, A., Lucas, C. G., Griffiths, T. L., \& Jacobs, L. (2011). A bayesian model of navigation in squirrels. In Proceedings of the 33rd annual meeting of the cognitive science society.

Waismeyer, A. S., \& Jacobs, L. F. (2013). The emergence of flexible spatial strategies in young children. Developmental Psychology, 49, 232.

Wystrach, A., Schwarz, S., Baniel, A., \& Cheng, K. (2013). Backtracking behaviour in lost ants: An additional strategy in their navigational toolkit. Proceedings of the Royal Society of 
London B: Biological Sciences, 280.

Yuille, A. L., \& Bülthoff, H. H. (1996). Bayesian decision theory and psychophysics. In D. C. Knill \& W. Richards (Eds.), Perception as Bayesian inference (pp. 123-162). Cambridge University Press.

Zhao, M., \& Warren, W. H. (2015). How you get there from here interaction of visual landmarks and path integration in human navigation. Psychological Science, 26, 915-924. 